ARTICLE

Received 24 Dec 2014 | Accepted 26 Apr 2015 | Published 18 Jun 2015

DOI: $10.1038 /$ ncomms 8313

\title{
Multivalency governs HP1 $\alpha$ association dynamics with the silent chromatin state
}

\author{
Sinan Kilic ${ }^{1}$, Andreas L. Bachmann', Louise C. Bryan \& Beat Fierz
}

Multivalent interactions between effector proteins and histone post-translational modifications are an elementary mechanism of dynamic chromatin signalling. Here we elucidate the mechanism how heterochromatin protein $1 \alpha(H P 1 \alpha)$, a multivalent effector, is efficiently recruited to the silent chromatin state (marked by trimethylated $\mathrm{H} 3$ at Lys9, H3K9me3) while remaining highly dynamic. Employing chemically defined nucleosome arrays together with single-molecule total internal reflection fluorescence microscopy (smTIRFM), we demonstrate that the HP1 $\alpha$ residence time on chromatin depends on the density of H3K9me3, as dissociated factors can rapidly rebind at neighbouring sites. Moreover, by chemically controlling HP1 $\alpha$ dimerization we find that effector multivalency prolongs chromatin retention and, importantly, accelerates the association rate. This effect results from increased avidity together with strengthened nonspecific chromatin interactions of dimeric HP1 $\alpha$. We propose that accelerated chromatin binding is a key feature of effector multivalency, allowing for fast and efficient competition for binding sites in the crowded nuclear compartment.

\footnotetext{
${ }^{1}$ Laboratory of Biophysical Chemistry of Macromolecules, Institute of Chemical Sciences and Engineering, Ecole Polytechnique Fédérale de Lausanne (EPFL), 1015 Lausanne, Switzerland. Correspondence and requests for materials should be addressed to B.F. (email: beat.fierz@epfl.ch).
} 
C hromatin effectors, carrying histone post-translational modification (PTM) binding domains (readers), are recruited to specific chromatin states where they alter chromatin structure, control gene expression or maintain genome integrity ${ }^{1,2}$. Dissociation constants for the interaction of individual reader domains with their cognate histone PTMs are relatively weak-often in the micromolar $(\mu \mathrm{M})$ range $^{1}$. Several models are conceivable to explain how chromatin effectors, despite their modest affinity, achieve efficient chromatin localization: First, the spatial co-existence of many binding sites in chromatin fibres allows rapid rebinding after dissociation. Second, oligomerization of effectors along the chromatin fibre can result in the formation of stable and long-lived complexes. Third, effectors often contain several binding domains or exist in homo- or hetero-oligomeric complexes. This allows simultaneous participation of several PTMs in multivalent binding interactions, resulting in strongly increased affinity. For most effectors it is, however, not known to which degree these putative mechanisms contribute to efficient recruitment to chromatin.

Here, we investigate the relevance of each of these proposed models for the mechanism of chromatin recruitment of $\mathrm{HP} 1 \alpha$, a multivalent effector. Proteins of the HP1 family are structural effectors that bind $\mathrm{H} 3 \mathrm{~K} 9 \mathrm{me} 3$ (ref. 3) and are involved in chromatin compaction ${ }^{4}$, gene repression, regulation of viral latency ${ }^{5}$ and control of transposable elements ${ }^{6}$. Of the three human HP1 isoforms ( $\alpha, \beta$ and $\gamma$ ), HP1 $\alpha$ and $\beta$ are associated with stably repressed heterochromatin at telomeres and centromeres, regions that are visible as dense chromatin foci in mammalian cells ${ }^{7}$. HP1 proteins bind to $\mathrm{H} 3 \mathrm{~K} 9 \mathrm{me} 2 / 3$ via an $\mathrm{N}$-terminal chromodomain (CD) with a dissociation constant $\left(K_{\mathrm{D}}\right)$ of $1-10 \mu \mathrm{M}$, depending on the subtype and species ${ }^{8-10}$. Further, in the S. pombe HP1 homologue Swi6, the CD has been found to form higher-order oligomers on chromatin binding ${ }^{11,12}$. In addition to the CD, HP1 contains a chromoshadow domain (CSD) responsible for protein dimerization ${ }^{13,14}$ and interaction with other proteins through PxVxL, PxVxI or related motifs ${ }^{15,16}$. A flexible, positively charged hinge region connects the $\mathrm{CD}$ and CSD and binds non-specifically to DNA as well as to RNA ${ }^{17,18}$, and these interactions have been implicated in heterochromatin maintenance ${ }^{17,19}$. In the nucleus, heterochromatin foci are stable over cell generations but exhibit fast dynamics on the individual protein level with HP1 residence times in the milliseconds-toseconds timescale ${ }^{20,21}$. Moreover, HP1 is quickly released from chromatin sites in response to $\mathrm{H} 3 \mathrm{~S} 10$ phosphorylation during mitosis ${ }^{22,23}$, as a reaction to DNA damage ${ }^{24}$, and during the repression of heterochromatic transcripts ${ }^{18}$. It is not well understood how HP1 $\alpha$ is efficiently recruited to heterochromatin sites enacting stable gene silencing, while maintaining a highly dynamic complex.

To investigate the recruitment and exchange dynamics of HP1 to modified chromatin fibres, highly sensitive experimental methods are required. Recent progress has been achieved in engineering synthetic chromatin fibres ${ }^{25}$ and in developing single-molecule techniques to analyse molecular ensembles in vitro ${ }^{26}$. Here we establish a fully chemically defined assay system to directly observe the real-time interaction dynamics of individual HP1 $\alpha$ molecules with their target histone PTMs within their native context, nucleosome arrays. This approach is highly complementary to measurements of chromatin effector dynamics in cells, as we have complete control over the chromatin architecture and modification status. The methodology allows us to monitor stochastic HP $1 \alpha$-chromatin interaction events in equilibrium. A high local $\mathrm{H} 3 \mathrm{~K} 9 \mathrm{me} 3$ density results in a prolonged $\mathrm{HP} 1 \alpha$ residence time due to rapid rebinding on dissociation. Employing a novel chemical strategy to control the dimeric state of HP1 $\alpha$, we discover that the increased avidity of a multivalent effector not only further prolongs its residence time, but also that it results in accelerated chromatin association. Importantly, we also observe this behaviour in the presence of PxVxI motifcontaining interaction partners, which stabilize the HP1 $\alpha$ dimeric state. High affinity of $\mathrm{HP} 1 \alpha$ for $\mathrm{H} 3 \mathrm{~K} 9 \mathrm{me} 3$-modified chromatin thus results from a fast chromatin association rate, while rapid local dissociation and rebinding kinetics ensure the maintenance of the dynamic complex required for heterochromatin function.

\section{Results}

HP1 $\alpha$ dynamically binds $\mathrm{H} 3 \mathrm{~K} 9 \mathrm{me} 3$ in chromatin. We set out to investigate $\mathrm{HP} 1 \alpha$ binding to $\mathrm{H} 3 \mathrm{~K} 9 \mathrm{me} 3$ carrying chromatin fibres by smTIRFM, employing a chemically defined model system (Fig. 1a). First, we required access to chromatin fibres containing $\mathrm{H} 3 \mathrm{~K} 9 \mathrm{me} 3$. We thus synthesized $\mathrm{H} 3 \mathrm{~K} 9 \mathrm{me} 3$ by traceless expressed protein ligation (EPL) ${ }^{27-29}$ (Fig. 1b and Supplementary Fig. 1) and reconstituted $\mathrm{H} 3 \mathrm{~K} 9 \mathrm{me} 3$-containing or unmodified histone octamers using recombinant human histones (Supplementary Fig. 2). In parallel, we generated a DNA template for chromatin array reconstitution. To this end, recombinant DNA containing 12 copies of the 177 base-pair '601' nucleosome positioning sequence $\mathrm{e}^{30,31}$ was ligated to a small oligonucleotide carrying a fluorescent dye (Atto647N) for localization, as well as biotin for immobilization on a solid support (Fig. 1c and Supplementary Fig. 3). Together with histone octamers containing either unmodified $\mathrm{H} 3$ or $\mathrm{H} 3 \mathrm{~K} 9 \mathrm{me} 3$, chromatin fibres were reconstituted following established protocols ${ }^{32}$ (Supplementary Figs 2 and 3). These chromatin arrays were then specifically immobilized on poly-ethylene glycol (PEG)-passivated coverslips ${ }^{33}$ by biotinneutravidin anchoring and imaged on a single-molecule level (Fig. 1d). To verify that the chromatin arrays remained intact on immobilization, we produced arrays with incorporated Atto488labelled H2A. The Atto488 fluorescence emission co-localized with Atto647N-labelled DNA and no histone loss was detected over the time of a typical experiment $(>1 \mathrm{~h}$ ) (Fig. 1e), indicating that the arrays were stable under single-molecule conditions.

Second, to detect individual binding events we had to label HP1 $\alpha$ with suitable fluorophores. To minimize structural perturbations of the protein, we decided to employ an EPL-based C-terminal labelling strategy. In a one-pot reaction, recombinant $\mathrm{HP} 1 \alpha$ fused to the N-terminal part of the N. punctiforme (Npu) split-intein was converted into a C-terminal thioester ${ }^{34}$ followed by in situ ligation to a tripeptide carrying an Atto532 fluorophore (P1, Fig. 2a and Supplementary Fig. 4), resulting in a labelling efficiency of up to $75 \%$. The dissociation constant $\left(K_{\mathrm{D}}\right)$ of the labelled $\mathrm{HP} 1 \alpha$ and a K9me3 containing $\mathrm{H} 3$ peptide was determined to be $5.36 \pm 1.33 \mu \mathrm{M}$, similar to unlabelled $\mathrm{HP} 1 \alpha$ (Supplementary Fig. 5) and in agreement with literature values ${ }^{4}$.

Having all necessary components in hand, we then proceeded to measure HP1 $\alpha$-chromatin interaction dynamics using smTIRFM. To this end, $1 \mathrm{nM}$ Atto532-labelled HP1 $\alpha$ was injected into flow-cells containing immobilized, H3K9me3-modified chromatin arrays. Positions of single chromatin arrays were detected by Atto647N emission, whereas HP1 $\alpha$ binding dynamics were monitored by Atto532 emission (Fig. 2b), revealing rapid transient chromatin interactions (Fig. 2c). A kinetic analysis of the resulting dwell-time histograms revealed double-exponential dissociation kinetics, with a fast major phase decaying with a time constant of $\tau_{\text {off }, 1}=0.25 \pm 0.03 \mathrm{~s}$ ( \pm s.d.) and a slower process with $\tau_{\text {off }, 2}=2.30 \pm 0.88 \mathrm{~s}$ (Fig. $2 \mathrm{~d}$, for all measured kinetic constants, refer to Table 1). These time constants are comparable to HP1 $\alpha$ dwell times observed in living cells ${ }^{21}$. Furthermore, the observed dwell times were not limited by fluorophore photobleaching, as HP1 $\alpha$-bound Atto532 decayed with a time constant $>40$ s under typical imaging conditions (Supplementary Fig. 6). 
a

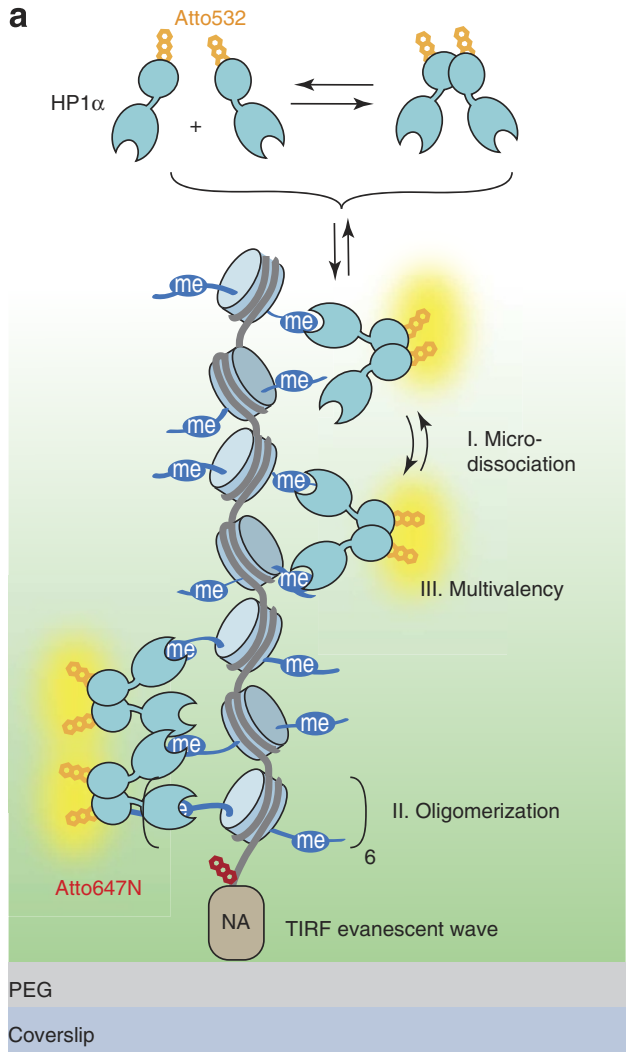

b

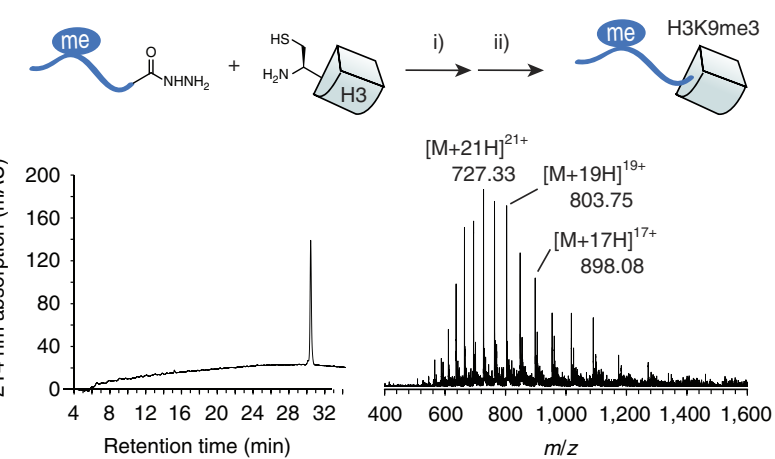

C

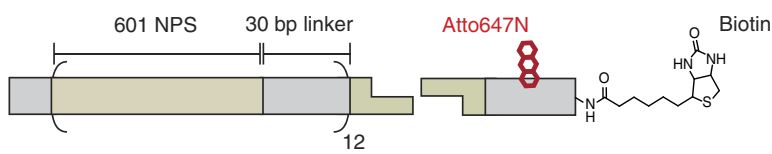

d

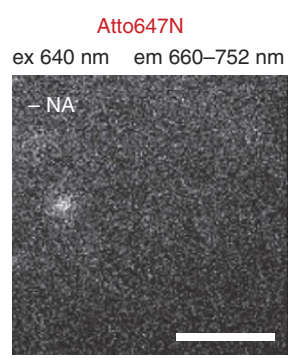

\section{e}

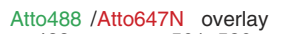
ex $488 \mathrm{~nm}$ em $501-520 \mathrm{~nm}$ $\begin{array}{ll}\text { ex } 488 \mathrm{~nm} & \text { em } 501-520 \mathrm{~nm} \\ \text { ex } 640 \mathrm{~nm} & \text { em 660-752 nm }\end{array}$

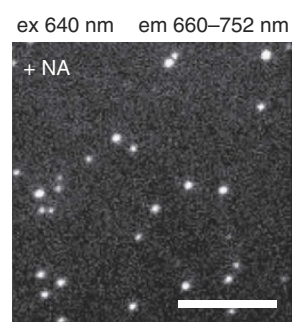

Figure 1 | An assay to measure effector interaction dynamics with chromatin arrays. (a) Scheme of the experiment: Atto532-labelled HP1 $\alpha$ dynamically interacts with immobilized H3K9me3-modified designer chromatin arrays, while the dynamic interactions are detected by smTIRFM. (b) Synthesis of H3K9me3 using a traceless EPL approach: (i) Oxidation of the hydrazide, in situ thioester formation and EPL reaction, (ii) desulfurization. Left: RP-HPLC analysis of the final protein, right: ESI-MS analysis of the final histone (MW: 15,251.8 Da calculated, 15,252 Da observed). (c) Design of chromatin DNA: recombinantly produced DNA carrying twelve 601-nucleosome position sequences (NPS) separated by 30 bp linkers, and a single, unique restriction site is ligated to a synthetic biotinylated and Atto647N-labelled oligonucleotide. (d) Chromatin array immobilization is specific and depends on neutravidin anchoring ( - NA, neutravidin absent, + NA, neutravidin present) as observed by Atto647N fluorescence emission. Scale bars, $5 \mu \mathrm{m}$. (e) Atto488-labelled $\mathrm{H} 2 \mathrm{~A}$, incorporated into the arrays, co-localizes with Atto647N-labelled DNA (orange spots).

Conversely, analysing the time intervals between binding events yielded an apparent association time constant of $\lambda_{\text {on }}=22.9 \pm 9.8 \mathrm{~s}$ (Fig. 2e). From $\lambda_{\text {on }}$ (taking into account the HP1 $\alpha$ concentration and the number of nucleosomes per array) a microscopic association rate constant of $k_{\mathrm{on}}=3.58 \times 10^{6} \mathrm{M}^{-1} \mathrm{~s}^{-1}$ was determined, resulting in an apparent $K_{\mathrm{D}}=1.1 \mu \mathrm{M}$ for a nucleosome in an array. This association rate constant is within the expected range of $10^{5}-10^{6} \mathrm{M}^{-1} \mathrm{~s}^{-1}$ for diffusion-controlled binding reactions of proteins $\mathrm{s}^{35}$. We then repeated the experiment using unmodified ( $\mathrm{H} 3 \mathrm{~K} 9 \mathrm{me} 0)$ chromatin arrays. In the absence of the mark, HP1 $\alpha$ binding was almost completely abolished, and the few binding events decayed with fast kinetics (Fig. 2f and Supplementary Fig. 7). In agreement, a CD mutant of HP1 $\alpha$ (W40A) exhibited very few binding events and fast off-rate constants, even in the presence of fully methylated arrays (Supplementary Fig. 7). These results indicate that we indeed directly measure histone PTM-dependent interactions while nonspecific binding events are rare.

HP1 $\alpha$ undergoes rapid dissociation and rebinding. Having established a system that allows the quantitative determination of the HP1 $\alpha$ interaction kinetics with modified chromatin arrays, we proceeded to test different models of interaction (see Fig. 1a). First, to assess the possibility that HP1 $\alpha$ undergoes a series of dynamic microdissociation and rebinding events, we investigated the kinetic effect of reducing the number of available binding sites. We thus assembled arrays containing different fractions of trimethylated H3K9. The stochastic binding dynamics of HP1 $\alpha$ to those arrays revealed that the residence time decreases with lower methylation density (Fig. 2f), indicating that rapid dissociation and rebinding events indeed contribute to the apparent residence time observed in densely modified chromatin. Moreover, we performed HP1 $\alpha$ binding experiments in the context of single nucleosomes, which also resulted in faster dissociation kinetics, due to the lack of neighbouring H3K9me3 sites (Supplementary Fig. 7).

HP1 $\alpha$ molecules compete for H3K9me3 sites. Second, we probed the ability of HP1 $\alpha$ to oligomerize along the chromatin fibre interactions, which should result in a prolonged retention of individual HP1 $\alpha$ proteins. Such a model for propagation of heterochromatin has been proposed for the yeast homologue Swi6 (refs 11,12). Under our standard measurement conditions the chromatin fibres are only occupied by single HP $1 \alpha$ monomers and dimers, thus rendering the formation of larger oligomers impossible. To increase HP1 $\alpha$ occupancy while retaining the possibility to observe single molecules, we therefore successively increased the total protein concentration in the flow cell by the addition of unlabelled HP1 $\alpha$. Contrary to our expectation, this resulted in a concentration-dependent decrease in $\tau_{\text {off }, 1}$, up to 3.5fold at $1 \mu \mathrm{M}$ total $\mathrm{HP} 1 \alpha$ concentration (Fig. $2 \mathrm{~g}$ ). This indicates 
a
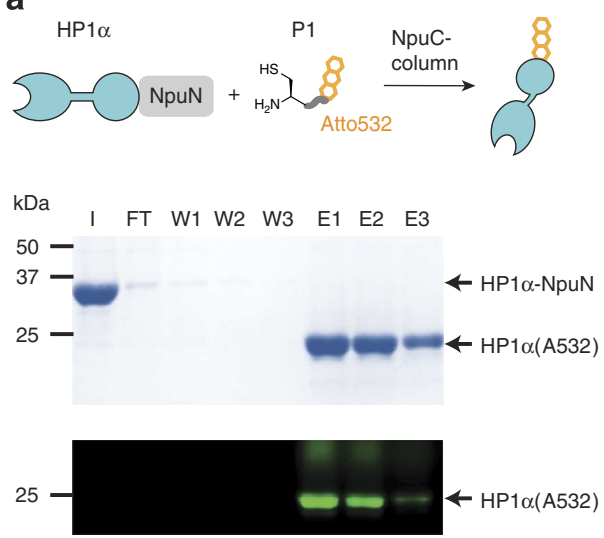

b

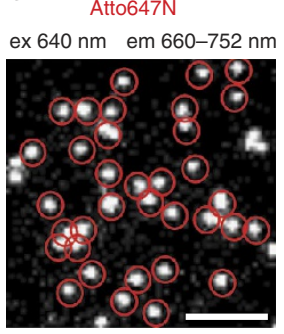

Atto532
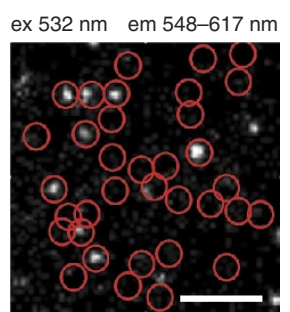

C
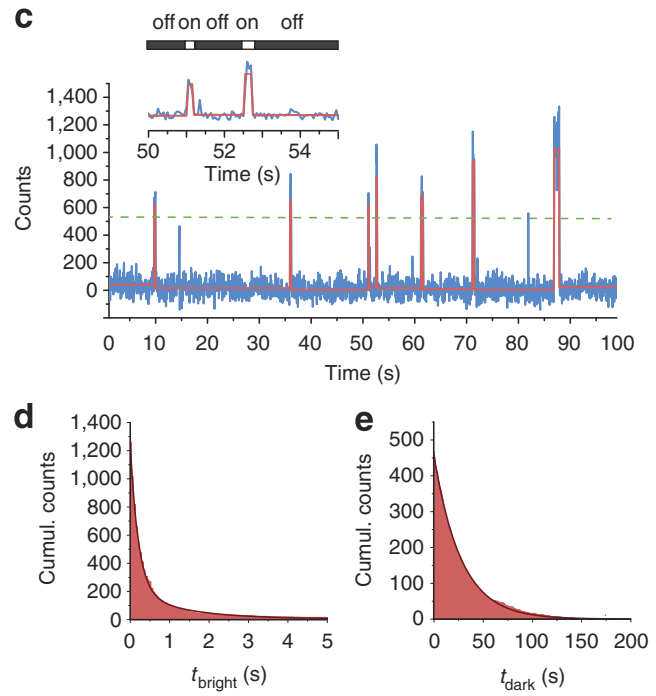

f
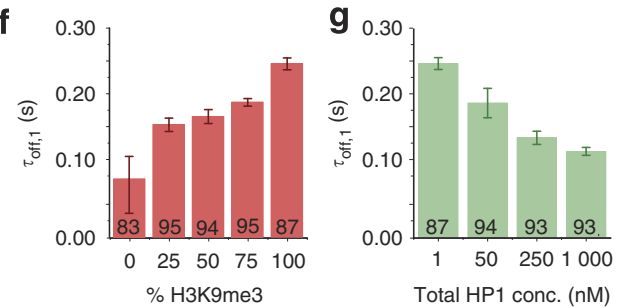

Figure 2 | HP1 $\alpha$ dynamically interacts with chromatin fibres. (a) HP1 $\alpha$ fused to NpuN is expressed, bound to NpuC-beads, cleaved in ligation buffer and reacted with P1 in situ, yielding labelled HP1 $\alpha$ (HP1 $\alpha($ A532)). ( I = input; FT = flowthrough; W1-3 = washes; E1-3 = elutions). (b) Left: single chromatin arrays are detected by Atto647N emission. Right: HP1 $\alpha$ interaction dynamics are monitored by Atto532 emission. Scale bar, $5 \mu \mathrm{m}$. (c) Time trace of fluorescence intensity (blue) from a single chromatin array showing transient HP1 $\alpha$ binding events, fitted by a step function (red). Inset: determination of bound (on-) and unbound (off-) times by a thresholding algorithm. (d) Dissociation kinetics: cumulative histogram of binding intervals ( $\left.t_{\text {bright }}\right)$ for 100 chromatin arrays $\left(10^{5}\right.$ frames each), fitted by a double-exponential function (fit: $\tau_{\mathrm{off}, 1}=0.25 \pm 0.03 \mathrm{~s}, \tau_{\mathrm{off}, 2}=2.26 \pm 1.22 \mathrm{~s}$ ). (e) Association kinetics: cumulative histogram of intervals between binding events ( $t_{\text {dark }}$ ) over 30 chromatin arrays, fitted by a single-exponential function (fit: $\lambda_{\text {on }}=22.9 \pm 9.8 \mathrm{~s}$ ). (f) The HP1 $\alpha$ residence time $\left(\tau_{\text {off }, 1}\right)$ depends on H3K9me3 density. Numbers indicate $\%$ amplitude of the fast phase (errors: s.e.m.; $n=2-16$ replicates). (g) The HP1 $\alpha$ residence time $\left(\tau_{\text {off, }, 1}\right)$ decreases as a function of HP1 $\alpha$ concentration due to local competition. The indicated concentration of unlabelled HP1 $\alpha$ is added to $1 \mathrm{nM}$ Atto532-labelled HP1 $\alpha$. Numbers indicate $\%$ amplitude of the fast phase (errors: s.e.m., $n=3-16$ replicates). (d-g) For all fit results, see Table 1.

that HP1 $\alpha$ does not predominantly form higher-oligomeric complexes, but rather that individual proteins compete for binding sites. This local competition then prevents rapid rebinding of dissociated HP1 $\alpha$ molecules, resulting in an apparent decrease in residence time, in a process termed facilitated dissociation $^{26,36}$. Such concentration-dependent dissociation kinetics were recently observed for several DNA-binding proteins $^{37,38}$.

Multivalent chromatin interactions accelerate HP1 $\alpha$ binding. Third, we explored the influence of multivalent chromatin interactions on $\mathrm{HP} 1 \alpha$ recruitment. $K_{\mathrm{D}}$ values of $0.5-5 \mu \mathrm{M}$ have been reported for CSD homodimerization of different HP1 subtypes $^{14}$. Therefore, under single-molecule conditions (at $1 \mathrm{nM}$ concentration) a large fraction of $\mathrm{HP} 1 \alpha$ dimers is dissociated into monomers. Indeed, an analysis of the fluorescence intensities of individual HP1 $\alpha$ binding events indicated that $17 \%$ of all observations could be attributed to dimeric proteins (Supplementary Fig. 9). First, we investigated whether disruption of any residual dimerization alters association dynamics. We therefore expressed and labelled an $\mathrm{HP} 1 \alpha$ protein with a mutated CSD (I163E), which can no longer form dimers ${ }^{13}$ (Supplementary Fig. 4). In this protein, the function of the $\mathrm{CD}$ was not impaired $\left(K_{\mathrm{D}}=9.4 \pm 2.8 \mu \mathrm{M}\right.$ for an $\mathrm{H} 3 \mathrm{~K} 9 \mathrm{me} 3$ peptide, Supplementary Fig. 5), and chromatin residence times were only slightly reduced as compared with wild-type HP1 $\alpha$ (Supplementary Fig. 7). In contrast, we observed an increased apparent association time $\lambda_{\text {on }}$ ( $>100 \mathrm{~s}$ ), demonstrating the requirement for CSD interactions for efficient chromatin binding.

Second, to constrain HP1 $\alpha$ in a dimeric state even under a low concentration regime, we developed a novel chemical strategy to produce covalently dimerized protein. In an affinity-directed dual-protein ligation reaction, two HP1 $\alpha$ protomers with C-terminal thioesters, which were optimally placed at the CSD, were ligated to a PxVxI-containing peptide with high CSD affinity and two ligation handles (Fig. 3a). The HP1-binding sequence of the shugoshin protein (hSgoL1, residues 448-457) ${ }^{39}$ was chosen as a suitable sequence. hSgoL1 plays a role in the protection of centromeric sister-chromatids and interacts with HP1, both in cell division and interphase with different functions ${ }^{39,40}$. We thus synthesized a peptide (P2), based on the hSgoL1 PxVxI-motif, flanked by two lysines with a cysteinyl-residue attached to their side chains, and containing the Atto532-fluorescent dye for detection (Fig. 3a). On the HP1 $\alpha$ CSD, installation of a C-terminal thioester at position 177 was assessed as being optimal for reactivity, and $\mathrm{HP} 1 \alpha(1-177)-\mathrm{SR}$ was prepared using the Npu split-intein. Subsequent in situ dual-ligation with $\mathbf{P 2}$ resulted in covalently linked dimers ( $\mathrm{HP} 1 \alpha_{\mathrm{cdm}}, 60 \%$ yield), which were further purified to homogeneity by size-exclusion chromatography (Fig. 3b and Supplementary Fig. 8). Finally, peptide binding assays revealed that the purified $H P 1 \alpha_{c d m}$ is 
Table 1 | Kinetic parameters of HP1 $\alpha$ - chromatin interaction dynamics.

\begin{tabular}{|c|c|c|c|c|c|c|c|c|c|}
\hline \multicolumn{2}{|c|}{ Experimental system } & \multicolumn{4}{|c|}{ Dissociation kinetics } & \multicolumn{2}{|c|}{ Association kinetics } & \multirow{2}{*}{$\begin{array}{l}\text { Equilibrium } \\
K_{\mathrm{d}}(\boldsymbol{\mu M}) \text { * }\end{array}$} & \multirow{2}{*}{$\begin{array}{c}\text { Replicates } \\
n\left(n^{\prime}\right) \dagger\end{array}$} \\
\hline Chromatin & Effector & $\tau_{\text {off, } 1}(\mathrm{~s})$ & $\% \mathbf{A 1}$ & $\tau_{\text {off, }, 2}(s)$ & $\% \mathbf{A 2}$ & $\lambda_{\text {on }}(\mathrm{s})$ & $\begin{array}{c}k_{\text {on }} \\
(M-1 \mathrm{~s}-1) \times 10^{6} \\
\end{array}$ & & \\
\hline H3K9me3 (100\%) & $\mathrm{HP} 1 \alpha$ & $0.25 \pm 0.03$ & $87 \pm 7$ & $2.26 \pm 1.22$ & $13 \pm 7$ & $22.9 \pm 9.8$ & $3.64 \pm 1.56$ & $1.16 \pm 0.54$ & $16(8)$ \\
\hline $\mathrm{H} 3 \mathrm{~K} 9 \mathrm{meO}$ & $\mathrm{HP} 1 \alpha$ & $0.08 \pm 0.03$ & $83 \pm 10$ & $(3.70 \pm 4.90) \ddagger$ & $17 \pm 10$ & $N / A^{\S}$ & $N / A^{\S}$ & $\mathrm{N} / \mathrm{A}^{\S}$ & $3(2)$ \\
\hline H3K9me3 (75\%) & $\mathrm{HP} 1 \alpha$ & $0.19 \pm 0.01$ & $95 \pm 3$ & $4.03 \pm 4.09$ & $5 \pm 3$ & $51.0 \pm 25.8$ & $1.63 \pm 0.82$ & $3.23 \pm 1.63$ & $5(3)$ \\
\hline H3K9me3 (50\%) & $\mathrm{HP} 1 \alpha$ & $0.17 \pm 0.03$ & $94 \pm 6$ & $4.09 \pm 3.62$ & $6 \pm 6$ & $48.2 \pm 15.2$ & $1.73 \pm 0.55$ & $3.40 \pm 1.24$ & $6(4)$ \\
\hline H3K9me3 (25\%) & $\mathrm{HP} 1 \alpha$ & $0.15 \pm 0.02$ & $95 \pm 2$ & $4.57 \pm 3.13$ & $5 \pm 2$ & $75.8 \pm 23.0$ & $1.10 \pm 0.33$ & $6.06 \pm 1.99$ & $6(3)$ \\
\hline H3K9me3 (MN) & $\mathrm{HP} 1 \alpha$ & $0.13 \pm 0.01$ & $78 \pm 11$ & $(1.18 \pm 0.62)^{\ddagger}$ & $22 \pm 11$ & $\mathrm{~N} / \mathrm{A}^{\S}$ & $N / A^{\S}$ & $N / A^{\S}$ & $3(3)$ \\
\hline H3K9me3 (100\%) & HP1 $1 \alpha+50$ nM comp. & $0.19 \pm 0.05$ & $94 \pm 3$ & $3.97 \pm 1.31$ & $6 \pm 3$ & $23.8 \pm 2.84$ & $3.55 \pm 0.42$ & $1.48 \pm 0.43$ & $5(3)$ \\
\hline H3K9me3 (100\%) & HP1 $1 \alpha+250$ nM comp. & $0.13 \pm 0.02$ & $93 \pm 2$ & $3.93 \pm 1.73$ & $7 \pm 2$ & $43.3 \pm 15.8$ & $2.07 \pm 0.62$ & $3.72 \pm 1.25$ & $3(2)$ \\
\hline H3K9me3 (100\%) & $\mathrm{HP} 1 \alpha+1000$ nM comp. & $0.11 \pm 0.01$ & $93 \pm 4$ & $2.75 \pm 0.72$ & $7 \pm 4$ & $29.3 \pm 5.51$ & $2.92 \pm 0.57$ & $3.11 \pm 0.67$ & $4(2)$ \\
\hline H3K9me3 (100\%) & HP1 $\alpha c d m$ & $0.33 \pm 0.01$ & $87 \pm 1$ & $3.40 \pm 0.53$ & $13 \pm 1$ & $7.45 \pm 1.87$ & $22.4 \pm 0.6^{11}$ & $0.13 \pm 0.01$ & $4(2)$ \\
\hline H3K9me3 (100\%) & HP1 $\alpha(1163 \mathrm{E})$ & $0.23 \pm 0.10$ & $85 \pm 7$ & $(4.11 \pm 2.83)^{\frac{1}{\ddagger}}$ & $15 \pm 7$ & $N / A^{\S}$ & $N / A^{\S}$ & $\mathrm{N} / \mathrm{A}^{\S}$ & $4(2)$ \\
\hline H3K9me3 (100\%) & $\mathrm{HP} 1 \alpha(\mathrm{W} 40 \mathrm{~A})$ & $0.10 \pm 0.02$ & $85 \pm 21$ & $(0.56 \pm 0.49)$ & $15 \pm 21$ & $\mathrm{~N} / \mathrm{A}^{\S}$ & $\mathrm{N} / \mathrm{A}^{\S}$ & $\mathrm{N} / \mathrm{A}^{\S}$ & $2(2)$ \\
\hline H3K9me3 (100\%) & $\mathrm{HP} 1 \alpha+\mathrm{P} 3$ & $0.33 \pm 0.04$ & $95 \pm 4$ & $4.80 \pm 3.96$ & $5 \pm 4$ & $10.5 \pm 0.59$ & $7.94 \pm 0.45$ & $0.38 \pm 0.05$ & $4(2)$ \\
\hline H3K9me3 (100\%) & $\mathrm{HP} 1 \alpha+\mathrm{P} 4$ & $0.28 \pm 0.01$ & $93 \pm 4$ & $4.45 \pm 2.10$ & $7 \pm 4$ & $19.6 \pm 6.52$ & $4.25 \pm 1.41$ & $0.84 \pm 0.28$ & $4(3)$ \\
\hline
\end{tabular}

The percentage numbers in brackets denote the H3K9me3 modification density. MN denotes mononucleosomes, $n$ is number of replicates.

${ }^{*} T h e K_{d} s$ are calculated based on the fast kinetic phase of the dissociation process.

$\dagger n$ indicates the number of independent HP1 $\alpha$ injections, $n^{\prime}$ indicates the number of flow cells used.

$\ddagger$ Due to insufficient statistics, $\tau_{\text {off }, 2}$ is poorly defined.

\$Due to insufficient statistics we cannot accurately determine association kinetics with $\lambda_{\text {on }}>100 \mathrm{~s}$

$\| k_{\text {on }}$ is calculated per molecule of $\mathrm{HP}_{\alpha, \mathrm{cdm}}$.
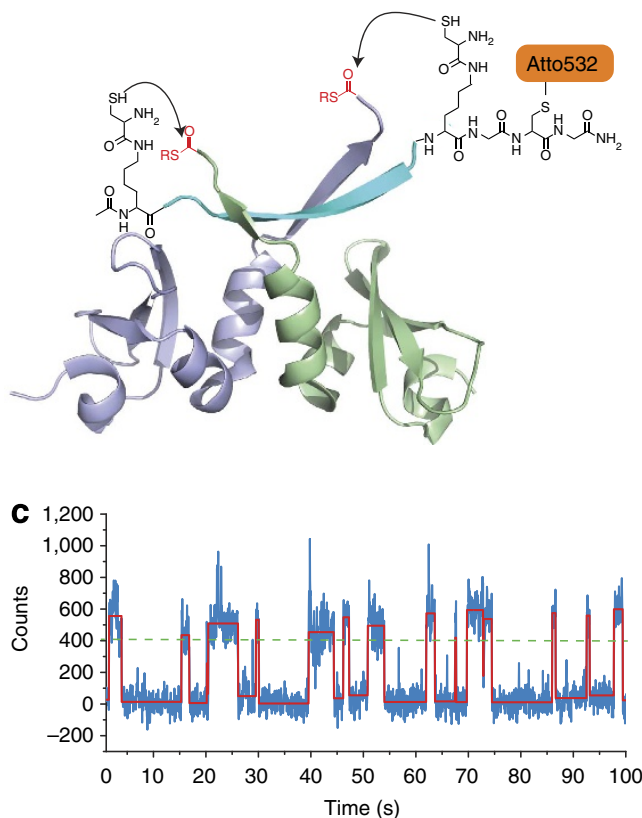

b
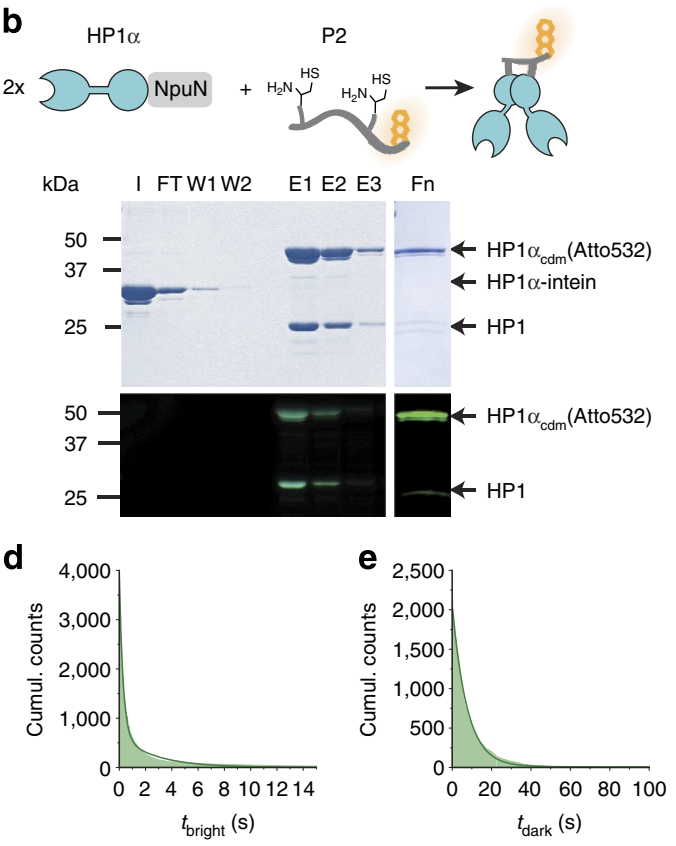

Figure 3 | Affinity-directed HP1 $\alpha$ dimerization allows investigating multivalent chromatin binding. (a) Strategy to synthesize HP1 $\alpha_{c d m}$ employing the PxVxI peptide $\mathbf{P 2}$ in an affinity-directed dual-EPL reaction (PDB: 3Q6S, ref. 39). (b) One-pot production of Atto532-labelled HP1 $\alpha_{c d m}$ : HP1 $\alpha$ (1-177), fused to the NpuN-split intein was bound to NpuC-beads and eluted with ligation buffer containing the hSgoL1 peptide (carrying two cysteinyl-lysines and an Atto532 dye), yielding labelled HP1 $\alpha_{c d m}$ (Atto532). Pure HP1 $\alpha_{c m d}$ was obtained after gel-filtration purification. Gel annotation: I=input; FT = flowthrough of column; W1-2 = column washes; E1-3= elution fractions; Fn= final protein after gel filtration. (c) Time trace of fluorescence intensity (blue) from a single chromatin array fitted by a step function (red) and showing transient HP1 $\alpha_{c d m}$ binding events (at $0.5 \mathrm{nM}$ ). (d) HP1 $\alpha_{c d m}$ dissociation kinetics (100 chromatin arrays, $10^{5}$ frames each) fitted by a double-exponential function (fit: $\tau_{\text {off, } 1}=0.33 \pm 0.01 \mathrm{~s}, \tau_{\text {off, } 2}=3.40 \pm 0.53 \mathrm{~s}$ ). (e) HP1 $\alpha_{\text {cdm }}$ association kinetics over 30 chromatin arrays, fitted by a single-exponential function (fit: $\lambda_{\text {on }}=7.45 \pm 1.87 \mathrm{~s}$ ). (d,e) For all fit results, see Table 1.

functional and binds to an $\mathrm{H} 3 \mathrm{~K} 9 \mathrm{me} 3$ peptide with an affinity similar to $\mathrm{HP} 1 \alpha\left(K_{\mathrm{D}}=12.5 \pm 0.5 \mu \mathrm{M}\right.$, Supplementary Fig. 5).

We then proceeded to probe chromatin binding with $\mathrm{HP} 1 \alpha_{\mathrm{cdm}}$. At a concentration of $0.5 \mathrm{nM} \mathrm{HP} 1 \alpha_{\mathrm{cdm}}$ (equivalent to $1 \mathrm{nM} \mathrm{HP} 1 \alpha$ monomers), we observed binding events of longer average duration and of increased frequency compared with HP1 $\alpha$ (Fig. 3c). Analysing the intervals of bound and unbound $\mathrm{HP} 1 \alpha_{\mathrm{cdm}}$ revealed double-exponential dissociation kinetics (Fig. 3d), with 
$\tau_{\text {off }, 1}=0.33 \pm 0.01 \mathrm{~s}$ (87\% amplitude) and $\tau_{\text {off }, 2}=3.40 \pm 0.53 \mathrm{~s}$ (13\% amplitude), and single-exponential binding kinetics with $\lambda_{\text {on }}=7.45 \pm 1.87 \mathrm{~s}$ (Fig. $3 \mathrm{e}$ ).

To analyse the effect of HP1 $\alpha$ dimerization on a single-array level, we generated two-dimensional histograms of $\tau_{\text {off, } 1}$ and $\tau_{\text {off, } 2}$ versus $\lambda_{\text {on }}$, where each data point originated from the analysis of the kinetic trace originating from an individual chromatin array. Inspecting the histograms for $\mathrm{HP} 1 \alpha$ (Fig. 4a) and $\mathrm{HP} 1 \alpha_{\mathrm{cdm}}$ (Fig. 4b) we observed a shift of the distribution to longer residence times and shorter $\lambda_{\text {on }}$ for the dimeric protein, as well as significant variation between the individual chromatin fibres. This variation may arise from local heterogeneity in nucleosome array structure, including dynamic conformational transitions, influencing the HP1 interaction modes.

From these measurements, it can be concluded that HP1 $\alpha$ dimerization directly results in a significant increase in chromatin residence time (Fig. 4c). This effect can be attributed to the increased possibility of simultaneous engagement of two K9me3bearing $\mathrm{H} 3$-tails in the chromatin array, the inability of dimers dissociating into monomers, as well as an increased chance of rebinding upon transient dissociation for $\mathrm{HP} 1 \alpha_{\mathrm{cdm}}$. Importantly, the dimeric $H P 1 \alpha_{c d m}$ also exhibited significantly more rapid chromatin binding, with $k_{\text {on }}=(2.24 \pm 0.60) \times 10^{7} \mathrm{M}^{-1} \mathrm{~s}^{-1}$ (Fig. 4d). This corresponds to a sixfold increase in the association rate constant for $\mathrm{HP} 1 \alpha_{\mathrm{cdm}}$ compared with $\mathrm{HP} 1 \alpha$, and results in an apparent $K_{\mathrm{D}}$ of $0.14 \mu \mathrm{M}$, about 60 -fold increased affinity compared with isolated $\mathrm{H} 3$ peptides (Supplementary Fig. 5).

To gain further insight into the molecular mechanism of how multivalent binders can speed up chromatin recruitment, we performed computational modelling. To explore the kinetic processes governing individual binding events, we implemented a coarse-grained computational model ${ }^{36}$ (Supplementary Fig. 10 and Supplementary Methods). Simulated association kinetics from a Brownian dynamics simulation of monomeric or dimeric binders interacting with nucleosomal arrays could reproduce an increase in association rate constant for the dimeric factors, compared with monomers (fourfold, Fig. 4e). An analysis of the trajectories revealed that the increase in association speed for a
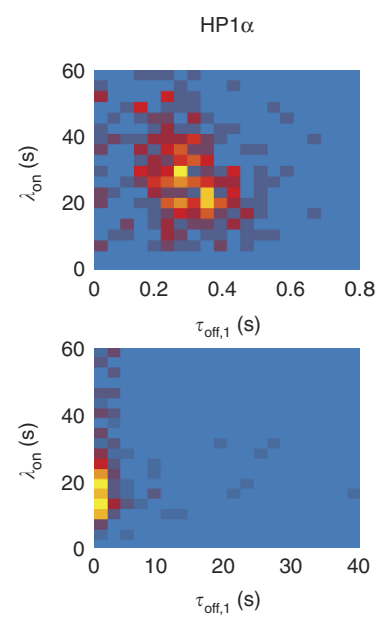

f

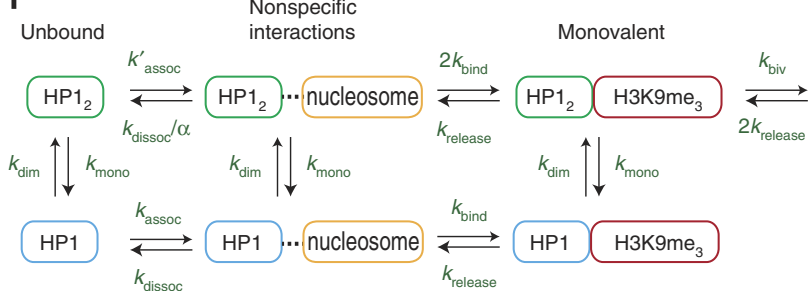

b
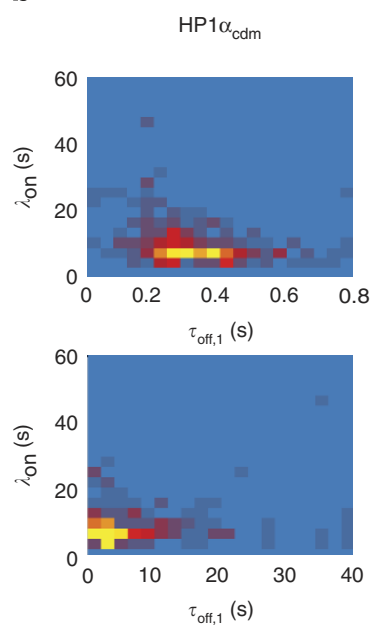

C
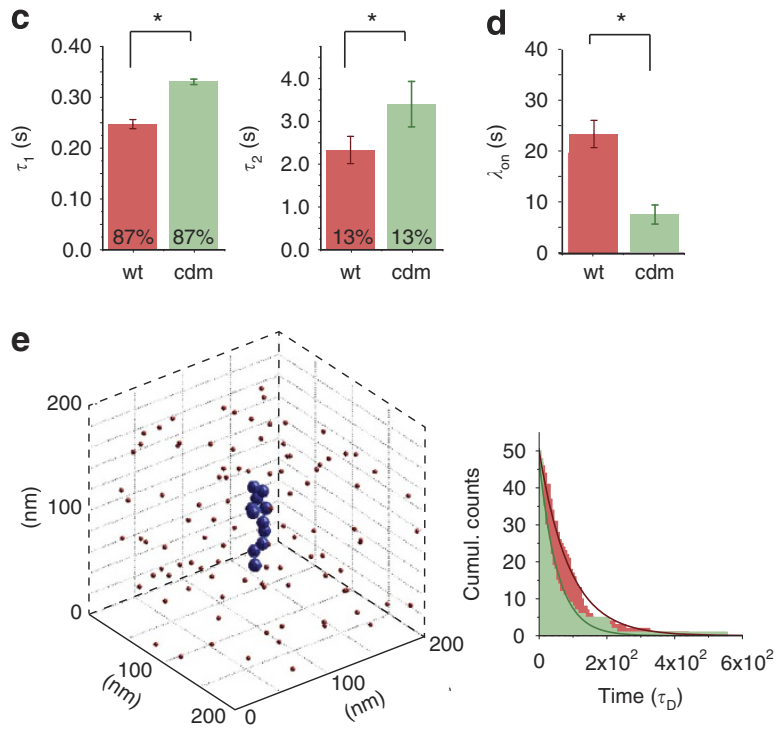

9

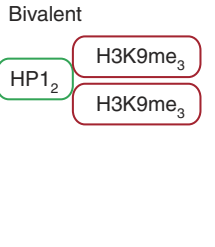

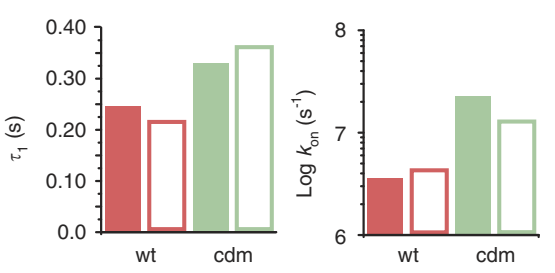

Figure 4 | Multivalent HP1 $\alpha$-chromatin interactions increase association speed. (a) Distribution of interaction kinetics from HP1 $\alpha$ with single chromatin arrays: Two dimensional histograms of single-array binding kinetics showing the correlation of dissociation time constants, $\tau_{\text {off, } 1}$ and $\tau_{\text {off, } 2}$ with the apparent association time constant $\lambda_{\text {on. }}$ (b) Two dimensional histogram of single-array binding kinetics for HP1 $\alpha_{c d m}$. (c) Comparison of the average dissociation time constants $\tau_{\text {off, } 1}$ and $\tau_{\text {off, } 2}$ (fast and slow phase) between HP1 $\alpha$ and HP1 $\alpha_{c d m}$ (errors: s.e.m., $n=4-16$ replicates, ${ }^{\star} P<0.05$, Student's t-test). (d) Comparison of the association rate constant $k_{\text {on }}$ between HP1 $\alpha$ and HP1 $\alpha_{c d m}$ (errors: s.e.m., $n=4-16$ replicates, ${ }^{\star} P<0.05$, Student's $t$-test). (c,d) For all fit results, see Table 1. (e) Brownian dynamics simulation of chromatin binding: monomeric or dimeric binders are diffusing in a box with $200 \mathrm{~nm}$ edge length containing a 12-nucleosome chromatin array. First passage binding kinetics are plotted for monomeric (red) and dimeric (green) binders at 20 and $10 \mu \mathrm{M}$ binder concentration, respectively. Single-exponential fits yield $\lambda_{\text {on }}=53.6 \tau_{\mathrm{D}}$ for the dimeric and $\lambda_{\text {on }}=89 \tau_{\mathrm{D}}$ for the monomeric binder $\left(\tau_{\mathrm{D}}\right.$ is the characteristic diffusion time, $\tau_{\mathrm{D}}=6 \pi \eta a^{3} /\left(k_{\mathrm{B}} T\right)$ for binders with radius $a$ in solvent with viscosity $\eta, k_{\mathrm{B}} T$ being the thermal energy). (f) Kinetic model for chromatin binding of HP1 $\alpha$ : HP1 $\alpha$ in a monomer-dimer equilibrium $\left(k_{\text {monor }} k_{\text {dim }}\right)$ associates nonspecifically $\left(k_{\text {assoc }}\right)$ and reversibly $\left(k_{\text {dissoc }}\right)$ with nucleosomes (short-lived interactions with (i) the nucleosomal or linker DNA, (ii) the nucleosomal surface or (iii) different regions on histone tails) before transitioning to a specific, H3K9me3 bound state $\left(k_{\text {bind }}\right)$ with a 100 ms-residence time $\left(k_{\text {release }}\right)$. The nonspecific association of dimeric protein is prolonged by a factor $\alpha$, and it can further proceed to a bivalent state $\left(k_{\text {biv }}\right)$. (g) Comparison of experimental data of HP1 $\alpha$ and HP1 $\alpha_{c d m}$ chromatin binding with results from kinetic model in (f). For parameters, see Supplementary Table 1 . 
multivalent binders arises mainly from presenting multiple interaction domains, in combination with enhanced nonspecific chromatin interactions in the dimers. These nonspecific interactions increase the time dimeric binders spend in the vicinity of chromatin, thereby raising the probability of a productive encounter.

These findings then allowed us to formulate a kinetic model to describe our experimental data (Fig. 4f). Stochastic simulations based on this kinetic model reproduced the measured major kinetic phases well (Fig. 4g, for parameters, see Supplementary Table 1). In agreement with the Brownian dynamics simulation, a rapid pre-equilibrium of nonspecific $\mathrm{HP} 1 \alpha$-chromatin interactions results in increased association kinetics for the dimeric protein if the release rate constant $k_{\text {dissoc }}$ is reduced for the dimer $(\alpha=5)$. In addition, the obtained microscopic rate constants for bivalent binding revealed that, at a given time, only a small fraction of dimeric $\mathrm{HP} 1 \alpha$ simultaneously engages two $\mathrm{H} 3 \mathrm{~K} 9 \mathrm{me} 3$ marks.

Induced HP1 dimerization drives chromatin association. $\mathrm{PxVxI} / \mathrm{L}$ containing peptides have been shown to induce HP1 $\alpha$ dimerization ${ }^{41}$. Correspondingly, proteins interacting with the CSD have been implicated in stabilizing HP1-chromatin interactions ${ }^{16,42}$

Thus, we investigated whether interactions at the CSD influence the chromatin association dynamics of HP1 $\alpha$ in cells. To this end we measured fluorescence recovery after photobleaching (FRAP) curves for wild-type and two mutant HP1 $\alpha$ variants (I163E and $\mathrm{W} 174 \mathrm{~A}$ ) fused to the monomeric fluorescent protein mEos3.2 (ref. 43). While the I163E mutant protein is strictly monomeric, the W174A mutation disrupts the interaction between the CSD and $\mathrm{PxVxI} / \mathrm{L}$ ligands ${ }^{13}$ and therefore abolishes ligand-induced dimerization. Transfected into NIH 3 T3 fibroblasts, all proteins produced discernable heterochromatin foci, which matched to regions of dense chromatin as observed by DNA staining (Fig. 5a). FRAP measurements within heterochromatin foci revealed that the complete loss in dimerization with the I163E mutant resulted in highly impaired chromatin binding, whereas also the W174A mutant exhibited reduced affinity, faster dynamics and a larger mobile fraction compared with wild-type HP1 $\alpha$ (Fig. 5b,c). This reveals the importance of CSD interactions, which favour HP1 $\alpha$ dimerization as well as organize $\mathrm{HP} 1 \alpha$ into larger complexes, and thus are important for efficient chromatin recruitment and stable association.

On the basis of these results, we tested whether we could reproduce the effect of CSD binding proteins on HP1 $\alpha$ chromatin association kinetics in our in vitro system. We thus synthesized a further variant of the PxVxI sequence of hSgoL1 (448-457) (P3, which binds $\mathrm{HP} 1 \alpha$ with a $K_{\mathrm{D}}=110 \mathrm{nM}$, Supplementary Fig. 11), and measured HP1-chromatin interactions in the absence and presence of $1 \mu \mathrm{M}$ P3. The resulting interaction dynamics showed both an increased residence time and significantly accelerated association when P3 was present (Fig. 5d,e). In agreement, the fluorescence intensity distribution of individual observations in the smTIRF traces showed a 2.5 -fold increase in HP1 $\alpha$ dimerization ( $42 \%$ dimeric $\mathrm{HP} 1 \alpha)$ upon peptide addition (Supplementary Fig. 9). Importantly, a diminished effect on chromatin association kinetics was measured when a mutated peptide, P4, was added (same sequence as P3 with the PxVxI motif mutated to A, Supplementary Fig. 11, due to its inability to induce HP $1 \alpha$ dimerization. A remaining nonspecific effect on the observed kinetics most likely originates from direct DNA interaction of the peptides, due to their poly-cationic character. Thus, protein-protein interactions at the CSD, which induce HP1 $\alpha$ dimerization and thereby multivalency, result in prolonged retention and accelerated chromatin association.

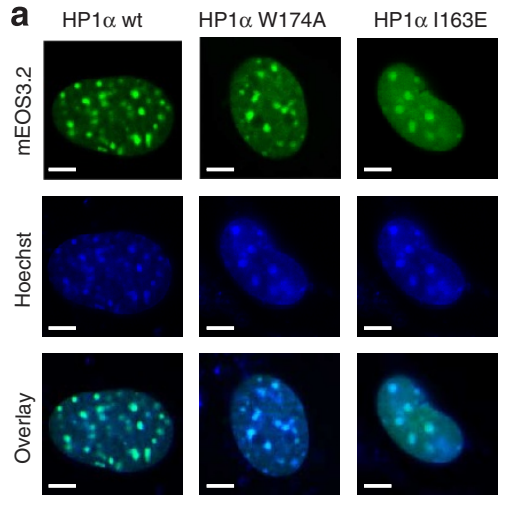

b $\mathrm{HP} 1 \alpha w \mathrm{wt}$

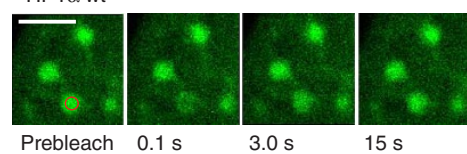

C
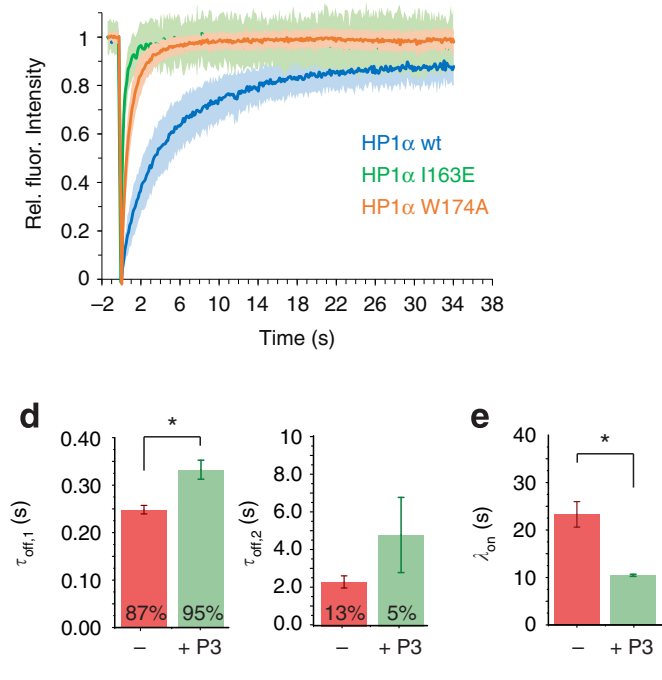

Figure 5 | CSD interactions accelerate HP1 association dynamics. (a) Confocal flurescence images of NIH 3T3 cells transfected with mEos3.2-HP1a, mEos3.2-HP1 $\alpha(1163 \mathrm{E})$ or mEos3.2-HP1 $\alpha(\mathrm{W} 174 \mathrm{~A})$ and overlay with Hoechst staining. Scale bar, $5 \mu \mathrm{m}$. (b) Time series of images from a typical FRAP

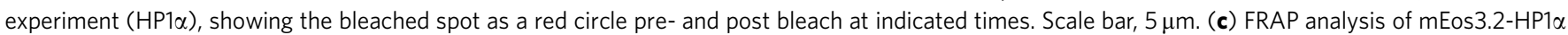
wt or W174A in heterochromatin foci ( $n=15$ cells with two foci from each, the shaded area denotes the standard deviation at each time point). Analysis of the traces using a diffusion/binding model ${ }^{46}$ results in the following values: HP1 $\alpha: \lambda_{\text {on }}=1.2 \pm 0.4 \mathrm{~s}$ and $\tau_{\text {off }}=2.8 \pm 1.2 \mathrm{~s}, \mathrm{HP} 1 \alpha(\mathrm{W} 174 \mathrm{~A}): \lambda_{\text {on }}=1.0 \pm 0.2 \mathrm{~s}$ and $\tau_{\text {off }}=1.1 \pm 0.3 \mathrm{~s}, \mathrm{HP} 1 \alpha(\mathrm{I} 163 \mathrm{E}): \lambda_{\text {on }}=1.8 \pm 0.3 \mathrm{~s}$ and $\tau_{\text {off }}=0.8 \pm 0.1 \mathrm{~s}$. (c) Comparison of the average dissociation time constants $\tau_{\text {off }, 1}$ and $\tau_{\text {off }, 2}$ (fast and slow phase) between HP1 $\alpha$ in the absence and presence of P3 (error bars: s.e.m., $n=4-16$ replicates, ${ }^{\star} P<0.05$, Student's $t$-test). (d) Comparison of the average dissociation time constants $\tau_{\text {off, } 1}$ and $\tau_{\text {off,2 }}$ (fast and slow phase) between HP1 $\alpha$ in the absence and presence of P3 (error bars: s.e.m., $n=4-16$ replicates, ${ }^{\star} P<0.05$, Student's $t$-test). (e) Comparison of the association rate constant $k_{\text {on }}$ between HP1 $\alpha$ in the absence and presence of $\mathbf{P} \mathbf{3}$ (errors: s.e.m., $n=4-16$ replicates, ${ }^{\star} P<0.05$, Student's t-test). (c,d) For all fit results, see Table 1. 


\section{Discussion}

Histone PTMs are read out by effector proteins or protein complexes, which often contain multiple reader domains. The increased avidity of these complexes allows multivalent engagement of multiple spatially confined marks in a chromatin fibre, thereby not only increasing the affinity towards a certain chromatin state but also providing a mechanism for combinatorial readout of co-existing histone PTMs ${ }^{44}$. Even when forming stable structures such as heterochromatin, chromatin effectors like HP1 $\alpha$ exist in a dynamic equilibrium between chromatin-bound states and an unbound pool of proteins ${ }^{20}$, thereby allowing a rapid response to external stimuli. However, how these effectors can be efficiently and stably recruited to a particular chromatin state while retaining fast exchange dynamics has remained an unsolved question. In living cells, FRAP and related photobleaching methods, as well as fluorescence correlation spectroscopy-based approaches have resulted in significant insight into the kinetic chromatin association processes for a large number of effectors ${ }^{45}$. However, a direct mechanistic interpretation is complicated by the fact that the chromatin state (amount, density and combinations of histone PTMs) at the observed nuclear binding sites is neither known nor can it be controlled in an experiment. Furthermore, the determination of kinetic parameters from FRAP or fluorescence correlation spectroscopy requires the application of mathematical models $^{46,47}$, which can lead to misinterpretations of the kinetics if not done carefully.

Here, we thus developed a general and complementary method, combining direct single-molecule detection with chemically defined chromatin fibres and purified effectors. This allowed us to directly observe interaction dynamics of single $\mathrm{HP} 1 \alpha$ molecules with H3K9 methylated chromatin arrays of controlled architecture and modification status. Furthermore, by employing chromatin arrays as binding substrate we could explore effects arising from the local accumulation of histone PTMs in their native chromatin environment. Our measurements of HP1 $\alpha$ dynamics revealed double-exponential dissociation kinetics whose major kinetic phase exhibited a residence time in the 100-300 ms range. The detected residence times were shown to depend on the density of $\mathrm{H} 3 \mathrm{~K} 9 \mathrm{me} 3$ on the chromatin arrays. This indicates that dissociation is not a monomolecular process but includes multiple microdissociation and rebinding events before final release. In addition, a slower process on the seconds timescale was detected, which may arise from multivalent interactions. In fact, HP1 $\alpha$ dynamics in living cells show a similar complex behaviour with multiscale kinetics ${ }^{21}$. Of note, in the cell, chromatin arrays are less homogenous compared with the model arrays used in the current study. Future studies are thus required to dissect how variability in the chromatin architecture (including variability in DNA sequence, linker DNA length or nucleosome occupancy) and internal chromatin dynamics influences effector binding kinetics.

Interestingly, raising the $\mathrm{HP} 1 \alpha$ concentration reduced the residence time of bound molecules in spite of increased chromatin occupancy. These findings argue against the presence of additional interactions between HP $1 \alpha$ molecules besides CSDmediated dimerization, and thus the formation of higher-order oligomers along the chromatin fibre is unlikely. Indeed, yeast Swi6, for which such a mechanism was proposed ${ }^{11,12}$, contains significant sequence differences compared with human HP1 $\alpha$, in particular in an extended $\alpha$-helix in its CD as well as in a lysinecontaining loop, which both were proposed to be an additional site of interaction. Rather, the data point towards a mechanism involving facilitated dissociation, resulting from competition for available binding sites, a common feature also recently described for DNA interacting proteins $26,36,37$.
Simultaneous interactions of dimeric HP1 $\alpha$ with two H3 tails have been proposed to induce chromatin compaction ${ }^{4}$, and such multivalency effects are expected to result in stable chromatin association of effectors in general ${ }^{48}$. To control the oligomeric state of $\mathrm{HP} 1 \alpha$ at the low concentration required for singlemolecule measurements, and thus to put these models to the test, we devised a new affinity-directed dual-ligation approach to covalently dimerize HP1 $\alpha$. Indeed, these molecules exhibited significantly faster association kinetics and longer residence times. Importantly, an increase in association kinetics could also be induced by the addition of a CSD-binding PxVxI-containing peptide derived from the HP1 $\alpha$-interacting protein hSgoL1. In agreement, CSD interacting proteins have been known to increase the equilibrium binding affinity of $\mathrm{HP} 1 \alpha^{42}$.

Taken together, our results suggest that the increased affinity upon HP1 $\alpha$ dimerization arises mainly through strengthening of the association rate constant. A strong increase in binding rate, as opposed to an effect on dissociation kinetics, allows to increase the local concentration of HP1 $\alpha$ at chromatin, while the complex remains very dynamic. In addition, in a crowded environment such as chromatin with high levels of competition for binding sites, fast binding kinetics are of critical importance ${ }^{35}$. Kinetic modelling provided a molecular explanation of the accelerated binding kinetics as a result from a strengthened non-specific encounter complex and increased probability of H3K9me3 binding for the dimeric molecule due to the presentation of two CDs. In contrast, we can exclude a direct allosteric activation of the CD through CSD dimerization, as both the monomeric $\mathrm{HP} 1 \alpha(\mathrm{I} 163 \mathrm{E})$ and the covalent dimeric HP1 $\alpha_{\mathrm{cdm}}$ exhibit comparable $\mathrm{H} 3 \mathrm{~K} 9 \mathrm{me} 3$ peptide affinities compared with $\mathrm{HP} 1 \alpha$ in equilibrium titration experiments.

In the cell, HP1 $\alpha$ exists at a concentration of around $0.5-1 \mu \mathrm{M}$ (ref. 21), depending on the chromatin compartment, which is close to the $K_{\mathrm{D}}$ of the CSD domains $(1.5-3 \mu \mathrm{M})^{14,49}$. Solely, on the basis of this value a significant monomeric fraction of HP $1 \alpha$ is expected. This fraction is, however, efficiently converted into dimeric and multivalent effectors upon interaction with binding partners associating in the CSD binding cleft. In agreement with this, our in vivo FRAP data show that $\mathrm{HP} 1 \alpha$ mutants carrying CSDs unable to interact with protein ligands are no longer able to stably associate with chromatin, presumably because they are unable to efficiently compete with wild-type HP1 molecules. Thus, the recruitment of HP1-interacting proteins represents an added layer of heterochromatin regulation through controlling multivalency and kinetics of $\mathrm{HP} 1 \alpha$ binding and exchange. Finally, HP1 $\alpha$ proteins are subject to PTMs including phosphorylation, acetylation $^{50}$ as well as SUMOylation ${ }^{51}$, which might alter protein-protein and protein-DNA interactions and further fine-tune the dynamic recruitment of $\mathrm{HP} 1 \alpha$. We expect that, in the competitive chromatin environment, effector binding kinetics critically determine the readout of single and combinatorial histone PTMs, and thus that effector multivalency may be a general strategy to control dynamic protein recruitment to chromatin.

\section{Methods}

EPL to generate H3 K9me3. Synthesis of H3 1-14 (K9me3) peptide hydrazide was performed on a pre-formed hydrazide resin using a standard fluorenylmethoxycarbonyl (FMOC) $\mathrm{N} \alpha$ protection strategy on an automated peptide synthesizer. In a typical ligation, $3 \mu \mathrm{mol}$ of $\mathrm{H} 31-14$ (K9me3) peptide hydrazide (5.0 mg) was dissolved in ligation buffer $(6 \mathrm{M} \mathrm{GdmCl}, 200 \mathrm{mM}$ phosphate, $\mathrm{pH} 3.0$ at $\mathrm{RT}$ ) to a final concentration of $5 \mathrm{mM}$ and the $\mathrm{pH}$ readjusted to 3.0. The peptide solution was kept at $-10^{\circ} \mathrm{C}$ in an ice/ $\mathrm{KCl}$ bath and $\mathrm{NaNO}_{2}$ was added dropwise from a $200 \mathrm{mM}$ stock solution to a final concentration of $15 \mathrm{mM}$. After mixing, the peptide was left at $-10^{\circ} \mathrm{C}$ for $20 \mathrm{~min}$. A total of $0.75 \mu \mathrm{mol}$ of the protein was dissolved in mercaptophenyl acetic acid (MPAA) ligation buffer $(6 \mathrm{M} \mathrm{GdmCl}$, $200 \mathrm{mM}$ phosphate, $300 \mathrm{mM}$ MPAA, $\mathrm{pH} 8.0$ ) to a final concentration of $1.2 \mathrm{mM}$. After 20 min of in situ activation the peptide was brought to room temperature and 
the solution of protein in MPAA ligation buffer mixed with the activated peptide. The $\mathrm{pH}$ was readjusted to 7.7-7.9 and ligation allowed to proceed until completion as determined by RP-HPLC (Supplementary Fig. 1c). When the reaction had reached completion, TCEP was added, the reaction was mixed with two volumes of RP-HPLC solvent $20 \%$ B and purified by semipreparative RP-HPLC using a gradient of $0-70 \% \mathrm{~B}$ over $45 \mathrm{~min}$. The ligation product was analysed by RP-HPLC and ESI-MS (Calculated MW $=15,283.9 \mathrm{Da}$, observed $\mathrm{MW}=15,285.0 \mathrm{Da}$ ) (Supplementary Fig. 1d).

Desulfurization to convert H3K9me3 A15C to H3K9me3. The ligation product was dissolved in TCEP desulfurization buffer $(6 \mathrm{M} \mathrm{GdmCl}, 200 \mathrm{mM}$ phosphate, $250 \mathrm{mM}$ TCEP, $\mathrm{pH} 6.5$ ) to a final concentration of $0.5 \mathrm{mM}$. Glutathione in TCEP desulfurization buffer and VA-044 radical starter were added from stock solutions to the ligation product to give final concentrations of 40 and $20 \mathrm{mM}$, respectively. The $\mathrm{pH}$ was readjusted to 6.5 and the desulfurization allowed to proceed at $37^{\circ} \mathrm{C}$. The reaction was continuously monitored by RP-HPLC and ESI-MS. When the reaction was complete, two volumes of RP-HPLC solvent (20\% B) were added and the product purified by semipreparative RP-HPLC using a gradient of $0-70 \%$ B over $45 \mathrm{~min}$. The protein was finally analysed by RP-HPLC and ESI-MS (Fig. 1b) (Calculated MW = 15,251.8 Da, observed $\mathrm{MW}=15,251.0 \mathrm{Da}$ ).

Histone octamer refolding. In a typical octamer refolding reaction, $0.4 \mathrm{mg}$ of each of the pure lyophilized human histones were dissolved in unfolding buffer (6 M GdmCl, $10 \mathrm{mM}$ Tris, $5 \mathrm{mM}$ DTT, $\mathrm{pH}$ 7.5). The exact concentration was determined by UV spectroscopy, using the following extinction coefficients: $\varepsilon_{280 \mathrm{~nm}, \mathrm{H} 2 \mathrm{~A}}=4,470 \mathrm{M}^{-1} \mathrm{~cm}^{-1}, \varepsilon_{280 \mathrm{~nm}, \mathrm{H} 2 \mathrm{~B}}=7,450 \mathrm{M}^{-1} \mathrm{~cm}^{-1}, \varepsilon_{280 \mathrm{~nm}, \mathrm{H} 3}=4,470$ $\mathrm{M}^{-1} \mathrm{~cm}^{-1}, \varepsilon_{280 \mathrm{~nm}, \mathrm{H} 4}=5,960 \mathrm{M}^{-1} \mathrm{~cm}^{-1}$. Equimolar amounts of $\mathrm{H} 3$ and $\mathrm{H} 4$ were then mixed along with 1.05 equivalents of $\mathrm{H} 2 \mathrm{~A}$ and $\mathrm{H} 2 \mathrm{~B}$ at $1 \mathrm{mg} \mathrm{ml}^{-1}$ and octamers were refolded by dialysis against refolding buffer $(2 \mathrm{M} \mathrm{NaCl}, 10 \mathrm{mM}$ Tris, $1 \mathrm{mM}$ EDTA, $5 \mathrm{mM}$ DTT, $\mathrm{pH}$ 7.5). The refolded octamers were subsequently purified by gel filtration on a Superdex S200 10/300GL column (Supplementary Fig. 2f,g). Collected fractions were analysed by sodium dodecyl sufate-PAGE (SDSPAGE), and octamer containing fractions were pooled and concentrated to $\sim 50 \mu \mathrm{M}$ octamer concentration. Finally, glycerol was added to a final concentration of $50 \%$, concentrations were determined by UV spectroscopy and octamer stocks were stored at $-20^{\circ} \mathrm{C}$.

\section{Generation of labelled and biotinylated chromatin array DNA. A plasmid} containing a $12 \times$ repeat of 601 nucleosome positioning sequence separated by 30-bp linker DNA segments (containing ScaI restriction sites) and a unique BsaI restriction site at the $3^{\prime}$ end was generated recombinantly, released from the vector backbone by EcorV digestion and purified by size-exclusion chromatography ${ }^{31}$. For labelling, $5 \mathrm{nmol}$ of a synthetic oligonucleotide ( $5^{\prime}$-ph-CAGCTAGTCTGCT- $3^{\prime}$ (amine-linker) 5'-CAGATATCGTCG-3'-Biotin), containing an internal amine attached to a dT and a C-terminal biotin, was mixed with $3 \mathrm{mM}$ A647N-NHS ester (from a $30 \mathrm{mM}$ stock in DMSO) in $0.1 \mathrm{M}$ sodium tetraborate and incubated overnight at $4{ }^{\circ} \mathrm{C}$. Subsequently, the labelled fragment was ethanol precipitated and the pellet was dissolved in $10 \mu \mathrm{l}$ of deionized miliQ water and quantified. Then, the labelled oligonucleotide was annealed to its complementary DNA strand (5'-CGACGATATCTGAGCAGACTA- $3^{\prime}$ ) in T4 DNA ligase reaction buffer (NEB). Subsequently, the labelled dsDNA oligonucleotide (5 eq. excess) was ligated to $12 \times 601$ array DNA using T4 DNA ligase for $1 \mathrm{~h}$ at room temperature (Supplementary Fig. 3a). The excess oligonucleotide was removed by purification with QIAquick spin columns (Qiagen), followed by PEG precipitation with $9 \%$ PEG 6000 (Supplementary Fig. 3a). Mononucleosome DNA was prepared using the same methodology, but employing a $1 \times 601$ NPS sequence. Concentrations and fluorescence were analysed by absorption spectrometry and fluorometry (Supplementary Fig. 3a).

Chromatin and nucleosome reconstitution. Nucleosomes and chromatin arrays were reconstituted on a scale from 10 to $100 \mathrm{pmol}$ (per mononucleosome) in 25-30 $\mu$ l. 601 NPS DNA ( 1 or 12 repeats) was reconstituted in TE buffer (10 mM Tris, $0.1 \mathrm{mM} \mathrm{EDTA}, \mathrm{pH} 7.5)$ and $2 \mathrm{M} \mathrm{NaCl}$, followed by the addition of 1.1 equivalents of the respective histone octamers. For chromatin arrays, 0.5 equivalents of MMTV buffer DNA was included ${ }^{31}$. The reactions were dialysed gradually from TEK2000 buffer (TE buffer including $2000 \mathrm{mM} \mathrm{KCl}$ ) to TEK10 buffer over $16 \mathrm{~h}$, using a two-channel peristaltic pump. After dialysis, chromatin concentrations were determined by UV quantification. $0.5-2$ pmol of chromatin arrays were digested with ScaI-HF (NEB) in $10 \mu \mathrm{l}$ for $6 \mathrm{~h}$. Quality of the reconstitution was assessed by native PAGE (Supplementary Fig. 3b,c). Chromatin and nucleosome reconstitutions were stored on ice for maximum 1 week after preparation.

HP1 $\alpha$ expression and labelling. Human $\mathrm{HP} 1 \alpha$ was expressed in E. coli as a C-terminal fusion to the $\mathrm{N}$-terminal half of the Npu split intein, followed by a hexahistidine tag. After purification by Ni-affinity and anion-exchange chromatography (to remove residual DNA), HP $1 \alpha-\mathrm{NpuN}$ was bound to a column of resin-linked NpuC. A $\mathrm{Thz}_{1}-\mathrm{G}_{2}-\mathrm{C}_{3}-\mathrm{CONH}_{2}$ tripeptide (P1) was synthesized by FMOC solid-phase peptide synthesis (Thz stands for thiazolidine). The peptide was subsequently reacted with Atto532-iodoacetamide in $20 \mathrm{mM}$ Tris buffer, $\mathrm{pH} 7.5$, followed by opening of Thz by incubation with $500 \mathrm{mM}$ methoxylamine at $\mathrm{pH} 5$. $1 \mathrm{mM}$ purified peptide was then added to the column-bound HP1 $\alpha$ in in situ EPL buffer (50 mM MPAA, $200 \mathrm{mM}$ MESNA, $100 \mathrm{mM}$ phosphate, $10 \mathrm{mM}$ TCEP, $1 \mathrm{mM}$ EDTA, pH 7.8). After overnight incubation at RT, the ligated protein was eluted, purified by size-exclusion chromatography, mixed with glycerol to $30 \%$ and flash frozen in small aliquots for later use.

HP1 $_{\text {cdm }}$ synthesis. HP1 $\alpha(1-177)-N p u N$ was expressed in E. coli and purified as described for HP1 $\alpha$-NpuN. The peptide P2, ac- $\mathrm{K}_{1} \mathrm{~S}_{2} \mathrm{~L}_{3} \mathrm{Y}_{4} \mathrm{P}_{5} \mathrm{~V}_{6} \mathrm{~V}_{7}$.

$\mathrm{K}_{8} \mathrm{I}_{9} \mathrm{R}_{10} \mathrm{R}_{11} \mathrm{~K}_{12} \mathrm{G}_{13} \mathrm{C}_{14} \mathrm{G}_{15}-\mathrm{CONH}_{2}$, was synthesized, using alloc-protecting groups for lysines 1 and 12. Following specific deprotection of the alloc group, N-Boc-Thz was coupled to the lysines, followed by peptide cleavage, purification, Atto532labelling using the iodoacetamide and final Thz opening by methoxylamine. The peptide was then added to intein-column-bound HP1 $\alpha(1-177)-\mathrm{NpuN}$ and reacted overnight at RT. The covalently dimerized HP1 was eluted and further purified by size-exclusion chromatography, mixed with glycerol to $30 \%$ and flash frozen in small aliquots for later use.

Microscale thermophoresis titrations. Measurements were carried out on a MonoLith NT.115 equipped with blue/green filters. H3K9me3 1-14 peptide from a main stock was serially diluted in titration buffer $(150 \mathrm{mM} \mathrm{NaCl}, 50 \mathrm{mM}$ HEPES, $0.05 \%$ Tween, $1 \mathrm{mM}$ DTT, $\mathrm{pH}=7.5$ ) to cover the concentration range $0.1-100 \mu \mathrm{M}$ and result in $10 \mu \mathrm{l}$ samples of each. From a $300 \mathrm{nM}$ stock (based on Atto532 absorption) of HP1 $\alpha$ (A532) or HP1 $\alpha_{\mathrm{cdm}}$ (A532), $10 \mu \mathrm{l}$ was added to the serially diluted peptide. Samples were loaded into hydrophilic-coated capillaries and cap scanning done at $100 \%$ LED power. Thermophoresis was done at $80 \%$ IR laser power. Data were normalized between the start and the end point and fitted with a quadratic binding equation using Origin.

Intrinsic tryptophan fluorescence titrations. A sample of $300 \mathrm{nM}$ unmodified HP1 $\alpha$ was loaded as $80 \mu \mathrm{l}$ into an Ultra-micro fluorescence cuvette. Increasing concentrations of $\mathrm{H} 3 \mathrm{~K} 9 \mathrm{me} 31-14$ peptide to cover the range $0.2-100 \mu \mathrm{M}$ was titrated in as $1.5-7 \mu \mathrm{l}$ per addition. Single-point measurements of the intrinsic tryptophan fluorescence were done for each titration point using $280 \mathrm{~nm}$ as excitation wavelength and $3 \mathrm{~nm}$ as the excitation slit width. Emission was measured at $348 \mathrm{~nm}$ with a slit width of $3.0 \mathrm{~nm}$ and an integration time of $0.5 \mathrm{~s}$. The typical number of counts obtained was $50-70 \mathrm{k}$. The number of trials allowed per point was 5 or until a maximum s.d. of $0.5 \%$ was reached. The fluorescence counts measured were corrected for the dilution upon titration of the peptide and then normalized between the start and the end point. The normalized data were fitted with a quadratic binding equation using Origin.

smTIRFM experiments. Glass coverslips and microscopy slides containing drilled holes were cleaned by sonication in 10\% alconox, acetone and ethanol, followed by treatment with a mixture of concentrated sulfuric acid to $30 \%$ hydrogen peroxide (3:1). The water-rinsed and dried coverslips and slides were then silanized using $2 \%$ (3-aminopropyl)triethoxysilane in acetone and assembled into flow cells, containing four channels each separated by double-sided adhesive tape. Pipette tips were inserted into the drilled holes and the channels were sealed with epoxy glue. A solution of $100 \mathrm{mg} \mathrm{ml}^{-1} \mathrm{mPEG}(5000)$-succinimidyl carbonate containg $1 \%$ biotin-mPEG-succinimidyl carbonate was infused into the channels and reacted for $3 \mathrm{~h}$, resulting in efficient passivation of all exposed surfaces in the channels. The channels were extensively washed using water and buffer T50 (10 mM Tris, $50 \mathrm{mM}$ $\mathrm{KCl}$ ) buffer before proceeding to experiments. For chromatin immobilization, $0.2 \mathrm{mg} \mathrm{ml}^{-1}$ neutravidin solution was infused using a high-precision syringe pump and incubated for $5 \mathrm{~min}$, followed by extensive washes with T50. Then, $500 \mathrm{pM}$ chromatin arrays in T50 buffer were injected into the neutravidin treated flow chamber for $5 \mathrm{~min}$, followed by a wash with T50 and imaging buffer $(50 \mathrm{mM}$ HEPES, $130 \mathrm{mM} \mathrm{KCl}, 10 \%$ glycerol, $2 \mathrm{mM}$ trolox, $0.005 \%$ tween- $20,3.2 \%$ glucose, glucose oxidase/catalase enzymatic oxygen removal system). Chromatin coverage was observed in the TIRF microscope (Nikon Ti-E) by fluorescent emission in the far-red channel upon excitation by a 640-nm laser line. Dynamic experiments were initiated by infusion of $1 \mathrm{nM}$ Atto532-labelled $\mathrm{HP} 1 \alpha$ or $0.5 \mathrm{nM} \mathrm{HP} 1 \alpha_{\mathrm{cdm}}$ in imaging buffer. All proteins were freshly diluted from a $100 \mathrm{nM}$ stock and immediately injected to avoid changes in concentration due to adsorption to tube walls. HP1 $\alpha$ dynamics were observed in the yellow/orange channel using a $530 \mathrm{~nm}$ laser line for excitation at $20 \mathrm{~W} / \mathrm{cm}^{2}$ using an EMCCD camera (Andor iXon) at 20 frames/s for a $25 \times 50 \mu \mathrm{m}$ area at a resolution of $160 \mathrm{~nm} /$ pixels. Every 200 frames, an image of the chromatin positions in the far-red channel was recorded for drift correction.

Data analysis. Movies of HP1 $\alpha$-chromatin interactions were recorded and converted into individual traces using a custom-made semi-automated Matlab (Mathworks) script: in short, after a global baseline-correction and drift-correction (using the far-red images interspersed at 200 frame intervals), individual chromatin array positions were determined using a peak-finding algorithm from the far-red images. Fluorescence intensity traces for each chromatin position were obtained by integrating over a circle of 2 pixel radius. Individual HP1 fluorescence peaks were 
included based on point-spread-function (PSF) and distance cutoffs. Kinetics were extracted from fluorescence traces using a semi-automated thresholding algorithm. Cumulative histograms were constructed from dark and bright intervals and fitted to sums of exponential functions.

Cell culture. NIH/3T3 mouse fibroblasts were cultured in $75 \mathrm{~cm}^{2}$ tissue culture flasks in DMEM/F-12 supplemented with $10 \%$ Newborn calf serum at $37^{\circ} \mathrm{C}$ in a water-saturated $5 \% \mathrm{CO}_{2}$ atmosphere. The day before transfection $1.5-2.0 \times 10^{5}$ cells were seeded onto $25-\mathrm{mm}$ round coverslips in six-well plates and grown overnight. On the day of transfection with a $70-90 \%$ level of confluency, the cells were transfected with $0.8 \mu \mathrm{g}$ of plasmid DNA per well using Effectene according to the manufacturer's protocol.

Microscopy and FRAP analysis. Microscopy was performed $24-32 \mathrm{~h}$ posttransfection using an inverted LSM 700 confocal microscope and a Plan apochromat $\times 63 / 1.4 \mathrm{NA}$ objective. The solid-state lasers at 405 and $488 \mathrm{~nm}$ were used for excitation. A short pass filter below $490 \mathrm{~nm}$ and a long pass filter beyond $490 \mathrm{~nm}$ were used for DAPI and mEos3.2 fluorescence, respectively. Images were acquired in $512 \times 512$ pixels with a $0.07 \mu \mathrm{m}$ pixel size, 12 -bit grey-scale depth, line averaging of 4 and a pixel dwell time of $6.30 \mu \mathrm{s}$. The laser power was set at $1.5-3.0 \%$, the master gain at $750-800$ and the digital gain at 1.5 using a pinhole size of $60.5 \mu \mathrm{m}$. FRAP bleaching and time-series images were acquired in 128-128 pixels with $0.07 \mu \mathrm{m}$ pixel size, 12-bit grey-scale depth and a pixel dwell time of $1.58 \mu$ s (scan time: $121 \mathrm{~ms}$ ). Master gain and digital gain were 750-800 and 1.5, respectively, with the pinhole set at $201 \mu \mathrm{m}$. A circular spot of 14 pixels $(1.04 \mu \mathrm{m})$ in diameter was used for bleaching. Twenty prebleach images were acquired before 10 iterations of a bleaching pulse at $80 \%$ laser power used and images were acquired for the subsequent $34 \mathrm{~s}$. Photobleaching during the time-series was corrected using the intensity in the bleach region relative to the entire acquisition region. The time-intensity acquisitions were normalized to the pre-bleach intensity and the first image after the bleach pulse. Results are averaged over 30-40 individual FRAP curves for the wild type and the mutants.

Statistics. The experimental values were compared using the two tailed Student's $t$-test. In the figures, statistical significance was indicated with an asterisk if $P<0.05$

\section{References}

1. Taverna, S. D., Li, H., Ruthenburg, A. J., Allis, C. D. \& Patel, D. J. How chromatin-binding modules interpret histone modifications: lessons from professional pocket pickers. Nat. Struct. Mol. Biol. 14, 1025-1040 (2007).

2. Kouzarides, T. Chromatin modifications and their function. Cell 128, 693-705 (2007).

3. Hediger, F. \& Gasser, S. M. Heterochromatin protein 1: don't judge the book by its cover! Curr. Opin. Genet. Dev. 16, 143-150 (2006).

4. Azzaz, A. M. et al. Human heterochromatin protein 1alpha promotes nucleosome associations that drive chromatin condensation. J. Biol. Chem. 289, 6850-6861 (2014)

5. du Chéné, I. et al. Suv39H1 and HP1gamma are responsible for chromatinmediated HIV-1 transcriptional silencing and post-integration latency. Embo J. 26, 424-435 (2007).

6. Freitag, M., Hickey, P. C., Khlafallah, T. K., Read, N. D. \& Selker, E. U. HP1 Is Essential for DNA Methylation in Neurospora. Mol. Cell 13, 427-434 (2004).

7. Nielsen, A. L. et al. Interaction with members of the heterochromatin protein 1 (HP1) family and histone deacetylation are differentially involved in transcriptional silencing by members of the TIF1 family. EMBO J. 18, 6385-6395 (1999).

8. Nielsen, P. R. et al. Structure of the HP1 chromodomain bound to histone H3 methylated at lysine 9. Nature 416, 103-107 (2002).

9. Lachner, M., O’Carroll, D., Rea, S., Mechtler, K. \& Jenuwein, T. Methylation of histone H3 lysine 9 creates a binding site for HP1 proteins. Nature 410, 116-120 (2001).

10. Bannister, A. J. et al. Selective recognition of methylated lysine 9 on histone $\mathrm{H} 3$ by the HP1 chromo domain. Nature 410, 120-124 (2001).

11. Canzio, D. et al. Chromodomain-mediated oligomerization of HP1 suggests a nucleosome-bridging mechanism for heterochromatin assembly. Mol. Cell 41, 67-81 (2011).

12. Canzio, D. et al. A conformational switch in HP1 releases auto-inhibition to drive heterochromatin assembly. Nature 496, 377-381 (2013).

13. Brasher, S. V. et al. The structure of mouse HP1 suggests a unique mode of single peptide recognition by the shadow chromo domain dimer. EMBO J. 19, 1587-1597 (2000).

14. Mendez, D. L. et al. The HPla disordered C terminus and chromo shadow domain cooperate to select target peptide partners. Chembiochem 12, 1084-1096 (2011)

15. Smothers, J. F. \& Henikoff, S. The HP1 chromo shadow domain binds a consensus peptide pentamer. Curr. Biol. 10, 27-30 (2000).
16. Thiru, A. et al. Structural basis of HP1/PXVXL motif peptide interactions and HP1 localisation to heterochromatin. EMBO J. 23, 489-499 (2004).

17. Muchardt, C. et al. Coordinated methyl and RNA binding is required for heterochromatin localization of mammalian HP1alpha. EMBO Rep. 3, 975-981 (2002).

18. Keller, C. et al. HP1(Swi6) mediates the recognition and destruction of heterochromatic RNA transcripts. Mol. Cell 47, 215-227 (2012).

19. Maison, C. et al. Higher-order structure in pericentric heterochromatin involves a distinct pattern of histone modification and an RNA component. Nat. Genet. 30, 329-334 (2002).

20. Cheutin, T. et al. Maintenance of stable heterochromatin domains by dynamic HP1 binding. Science 299, 721-725 (2003)

21. Muller, K. P. et al. Multiscale analysis of dynamics and interactions of heterochromatin protein 1 by fluorescence fluctuation microscopy. Biophys. J. 97, 2876-2885 (2009).

22. Hirota, T., Lipp, J. J., Toh, B.-H.H. \& Peters, J.-M.M. Histone H3 serine 10 phosphorylation by Aurora B causes HP1 dissociation from heterochromatin. Nature 438, 1176-1180 (2005).

23. Fischle, W. et al. Regulation of HP1-chromatin binding by histone $\mathrm{H} 3$ methylation and phosphorylation. Nature 438, 1116-1122 (2005).

24. Ayoub, N., Jeyasekharan, A. D., Bernal, J. A. \& Venkitaraman, A. R. HP1-beta mobilization promotes chromatin changes that initiate the DNA damage response. Nature 453, 682-686 (2008).

25. Fierz, B. \& Muir, T. W. Chromatin as an expansive canvas for chemical biology. Nat. Chem. Biol. 8, 417-427 (2012).

26. Ha, T. Single-molecule approaches embrace molecular cohorts. Cell 154, 723-726 (2013).

27. Muir, T. W., Sondhi, D. \& Cole, P. A. Expressed protein ligation: a general method for protein engineering. Proc. Natl Acad. Sci. USA 95, 6705-6710 (1998).

28. Yan, L. Z. \& Dawson, P. E. Synthesis of peptides and proteins without cysteine residues by native chemical ligation combined with desulfurization. J. Am. Chem. Soc. 123, 526-533 (2001).

29. Wan, Q. \& Danishefsky, S. J. Free-radical-based, specific desulfurization of cysteine: A powerful advance in the synthesis of polypeptides and glycopolypeptides. Angew. Chem. Int. Ed. Engl. 46, 9248-9252 (2007).

30. Lowary, P. T. \& Widom, J. New DNA sequence rules for high affinity binding to histone octamer and sequence-directed nucleosome positioning. J. Mol. Biol. 276, 19-42 (1998).

31. Dorigo, B., Schalch, T., Bystricky, K. \& Richmond, T. J. Chromatin fiber folding: requirement for the histone $\mathrm{H} 4 \mathrm{~N}$-terminal tail. J. Mol. Biol. 327, 85-96 (2003).

32. Fierz, B. et al. Histone H2B ubiquitylation disrupts local and higher-order chromatin compaction. Nat. Chem. Biol. 7, 113-119 (2011).

33. Roy, R., Hohng, S. \& Ha, T. A practical guide to single-molecule FRET. Nat. Methods 5, 507-516 (2008).

34. Vila-Perello, M. et al. Streamlined expressed protein ligation using split inteins J. Am. Chem. Soc. 135, 286-292 (2013).

35. Schreiber, G., Haran, G. \& Zhou, H. X. X. Fundamental aspects of proteinprotein association kinetics. Chem. Rev. 109, 839-860 (2009).

36. Sing, C. E., Olvera de la Cruz, M. \& Marko, J. F. Multiple-binding-site mechanism explains concentration-dependent unbinding rates of DNAbinding proteins. Nucleic Acids Res. 42, 3783-3791 (2014).

37. Graham, J., Johnson, R. \& Marko, J. Concentration-dependent exchange accelerates turnover of proteins bound to double-stranded DNA. Nucleic Acids Res. 39, 2249-2259 (2011).

38. Loparo, J. J., Kulczyk, A. W., Richardson, C. C. \& van Oijen, A. M. Simultaneous single-molecule measurements of phage T7 replisome composition and function reveal the mechanism of polymerase exchange. Proc. Natl Acad. Sci. USA 108, 3584-3589 (2011).

39. Kang, J. et al. Mitotic centromeric targeting of HP1 and its binding to Sgol are dispensable for sister-chromatid cohesion in human cells. Mol. Biol. Cell 22, 1181-1190 (2011).

40. Yamagishi, Y., Sakuno, T., Shimura, M. \& Watanabe, Y. Heterochromatin links to centromeric protection by recruiting shugoshin. Nature 455, 251-255 (2008).

41. Mendez, D. L. et al. The HP1a disordered C terminus and chromo shadow domain cooperate to select target peptide partners. Chembiochem. 12, 1084-1096 (2011).

42. Eskeland, R., Eberharter, A. \& Imhof, A. HP1 binding to chromatin methylated at H3K9 is enhanced by auxiliary factors. Mol. Cell Biol. 27, 453-465 (2007).

43. Zhang, M. et al. Rational design of true monomeric and bright photoactivatable fluorescent proteins. Nat. Methods 9, 727-729 (2012).

44. Musselman, C., Lalonde, M. -E., Côté, J. \& Kutateladze, T. Perceiving the epigenetic landscape through histone readers. Nat. Struct. Mol. Biol. 19, 1218-1227 (2012). 
45. Steffen, P. A., Fonseca, J. P. \& Ringrose, L. Epigenetics meets mathematics: towards a quantitative understanding of chromatin biology. Bioessays 34, 901-913 (2012).

46. Sprague, B. L., Pego, R. L., Stavreva, D. A. \& McNally, J. G. Analysis of Binding Reactions by Fluorescence Recovery after Photobleaching. Biophys. J. 86, 34733495 (2004).

47. Michelman-Ribeiro, A. et al. Direct measurement of association and dissociation rates of DNA binding in live cells by fluorescence correlation spectroscopy. Biophys. J. 97, 337-346 (2009).

48. Ruthenburg, A. J., Li, H., Patel, D. J. \& Allis, C. D. Multivalent engagement of chromatin modifications by linked binding modules. Nat. Rev. Mol. Cell Biol. 8, 983-994 (2007).

49. Müller-Ott, K. et al. Specificity, propagation, and memory of pericentric heterochromatin. Mol. Syst. Biol. 10, 746 (2014).

50. LeRoy, G. et al. Heterochromatin protein 1 is extensively decorated with histone code-like post-translational modifications. Mol. Cell Proteomics 8, 2432-2442 (2009).

51. Maison, C. et al. SUMOylation promotes de novo targeting of HP1 $\alpha$ to pericentric heterochromatin. Nat. Genet. 43, 220-227 (2011).

\section{Acknowledgements}

We thank Joachim Piquet, Horst Vogel and Daniel Ciepielewski (Nikon $\mathrm{CH}$ ) for help with setting up the TIRF microscope, Aleksandr Benke, Seamus J. Holden and Sulian Manley for help with initial experiments, Carolin Lechner for assistance with peptide synthesis, Horst Pick for help with cell culture, Arne Seitz and Thierry Laroche (BIOP, EPFL) for assistance with the FRAP experiments, Horst Vogel, Champak Chatterjee, Kai Johnsson and all members of LCBM for fruitful discussions and comments on the manuscript. For financial support we thank the Sandoz Family Foundation, the Swiss National Science Foundation (grant 31003A_149789) and EPFL. S.K. is kindly supported by a fellowship from the Boehringer Ingelheim Fonds.

\section{Author contributions}

S.K. and B.F designed the experiments with assistance from all co-authors. S.K., A.B. and B.F. performed peptide and protein chemistry, L.B., S.K. and B.F. produced DNA constructs. All authors performed biophysical measurements and analysed the data. S.K. performed the cell experiments. S.K. and B.F. wrote the manuscript with assistance from all co-authors.

\section{Additional information}

Supplementary Information accompanies this paper at http://www.nature.com/ naturecommunications

Competing financial interests: The authors declare no competing financial interests.

Reprints and permission information is available online at http://npg.nature.com/ reprintsandpermissions/

How to cite this article: Kilic, S. et al. Multivalency governs HP1 $\alpha$ association dynamics with the silent chromatin state. Nat. Commun. 6:7313 doi: 10.1038/ncomms8313 (2015).

This work is licensed under a Creative Commons Attribution 4.0 International License. The images or other third party material in this article are included in the article's Creative Commons license, unless indicated otherwise in the credit line; if the material is not included under the Creative Commons license, users will need to obtain permission from the license holder to reproduce the material. To view a copy of this license, visit http://creativecommons.org/licenses/by/4.0/ 